\title{
Importance of initial and final states as intermediate states in two-photon spectroscopy of polar molecules
}

\author{
Bernhard Dick a) and Georg Hohlneicher \\ Lehrstuhl für Theoretische Chemie der Universität zu Köln, Köln, West Germany \\ (Received 25 January 1982; accepted 18 February 1982) \\ Two-photon transitions may proceed via a mechanism not involving virtually excited intermediate states. For \\ some examples, it is shown numerically that this mechanism may account for the largest contribution to the \\ two-photon cross section in polar molecules, if the dipole moment strongly alters with excitation. Possible \\ consequences for the two-photon detection of "forbidden" states in polar polyene systems are discussed.
}

\section{INTRODUCTION}

During the 1970's, we have learned that in most unsaturated organic molecules, e.g., such as polyenes and polyacenes, the number of low lying electronically excited states which cannot be seen in the UV spectrum, due to their small or even vanishing dipole transition moment, is considerably larger than suggested earlier. ${ }^{1-3}$ This finding is not only an interesting spectroscopic fact which has changed the understanding of the electronic structure of these systems, but it also has important implications for the discussion of possible photochemical routes. It is, therefore, of ten desriable to derive more detailed information on the location and order of these "dipole forbidden states." 4 Due to often complementary selection rules, two-photon absorption (TPA) became one of the most important experimental tools for the investigation of dipole forbidden transitions (DFT). ${ }^{6.7}$ For the interpretation of measured TPA spectra and for the assignment of the observed bands, we usually need theoretical estimates for energies and cross sections of the corresponding transitions. Whereas the energies are basically obtained in the same theoretical framework as used for dipole allowed transitions, the calculation of the two-photon absorption cross sections involves an infinite sum over so-called "virtual intermediate states." Moreover, there exist different possible ways to perform this summation. ${ }^{8,9}$ These possibilities are necessarily equivalent, as long as the whole infinite sum is considered. In numerical applications, however, where the sum has to be truncated, the different formulas behave quite differently. In connection with the formula most suitable for these applications, there is some confusion in the literature as to whether the initial state $|i\rangle$ and the final state $|f\rangle$ have to be included in the summation as "intermediate states." Some authors do include $|i\rangle$ and $|f\rangle^{8,10,11}$ and some do not. ${ }^{12-14}$

It is the main purpose of this paper to show that the initial and final state have to be included, if the system under consideration has no center of symmetry and if the most suitable formula is used (Sec. II). In some cases those states may even become the most important intermediate states. This is demonstrated with a series of model compounds, all of which contain a

\footnotetext{
2) Present address: Department of Chemistry, University of Pennsylvania, Philadelphia, Pennsylvania 19104.
}

linear $6 \pi$-electron system (Sec. III). The results obtained in this section will show that great care has to be taken in the interpretation of TPA spectra of highly polar molecules, as, e.g., model compounds for the visual chromophor. ${ }^{15}$

\section{THEORY OF THE TWO-PHOTON TENSOR}

Second order time dependent perturbation theory, using the perturbation operator

$$
H^{\prime}=p \cdot A
$$

yields the following expression for the cross section of a two-photon transition $i \rightarrow f$ (the same nomenclature is used as in Ref. 8)

$$
\delta^{i f}=\left(\frac{e}{m}\right)^{4} \frac{4 \pi^{2} g\left(\omega_{1}+\omega_{2}\right)}{c^{2} \omega_{1} \omega_{2}}\left|e_{1} M^{i f} e_{2}\right|^{2}
$$

In Cartesian coordinates $[\alpha, \beta \epsilon(x, y, z)]$, the second rank tensor $\boldsymbol{M}^{\text {if }}$ has the following components:

$$
M_{\alpha \beta}^{i f}=\sum_{k}\left\{\frac{P_{i k}^{\alpha} P_{k f}^{\beta}}{E_{i k}-\hbar \omega_{1}}+\frac{P_{i k}^{\beta} P_{k f}^{\alpha}}{E_{i k}-\hbar \omega_{2}}\right\} .
$$

The summation index runs over all eigenstates of the system. Using the identity

$$
\begin{aligned}
\mathbf{p}_{a b} & =\frac{i m}{\hbar}\left(E_{a}-E_{b}\right)\langle a|\mathbf{r}| b\rangle \\
& =\frac{i m}{\hbar} E_{b a} \mathbf{R}_{a b},
\end{aligned}
$$

the matrix elements of the momentum operator in Eq. (3) can be replaced by matrix elements of the dipole operator

$$
\begin{aligned}
\tilde{S}_{\alpha \beta}^{i f} & =-\frac{\hbar^{2}}{m^{2}} M_{\alpha \beta}^{i f}=\sum_{k} E_{i k} E_{k f}\left\{\frac{R_{i k}^{\alpha} R_{k f}^{\beta}}{E_{i k}-\hbar \omega_{1}}+\frac{R_{i k}^{\beta} R_{k f}^{\alpha}}{E_{i k}-\hbar \omega_{2}}\right\} \\
& =\sum_{k} E_{i k} E_{k f} X_{k, i f}^{\alpha \beta} .
\end{aligned}
$$

Due to $E_{i j}=E_{f f}=0$ the initial and the final state do not contribute to $M$ if $\mathrm{Eq}$. (5) is used to calculate the components of the tensor $M$. However, as we have shown in Ref. 8, Eq. (5) exhibits very bad convergence if applied to numerical calculations where the sum over $k$ has to be truncated. It is, therefore, preferable to use another formula for $\tilde{\mathbf{s}}$ in which the unfavorable energy factor $E_{i k} \cdot E_{k f}$ (responsible for the bad convergence) is replaced by the product of the photon energies ${ }^{9}$ 


$$
\tilde{S}_{\alpha \beta}^{i f}=\hbar \omega_{1} \hbar \omega_{2} \sum_{k} X_{k, 1 f}^{\alpha \beta} .
$$

If Eq. (6) is used instead of Eq. (5), it is essential to retain the states $|i\rangle$ and $|f\rangle$ in the sum over $k$. This is shown by the following proof:

The matrix element of the commutator

$$
\left[r_{\alpha}, p_{B}\right]=i \hbar \delta_{\alpha \beta}
$$

between eigenstates $|i\rangle$ and $|f\rangle$ is

$$
\left\langle i\left|r_{\alpha} p_{\beta}\right| f\right\rangle-\left\langle i\left|p_{\beta} r_{\alpha}\right| f\right\rangle=i \hbar \delta_{\alpha \beta} \delta_{i f} .
$$

Resolution on the identity

$$
1=\sum_{h}|k\rangle\langle k|
$$

leads to

$$
\sum_{k}\left\{R_{i k}^{\alpha} P_{k f}^{\beta}-P_{i k}^{\beta} R_{k f}^{\alpha}\right\}=i \hbar \delta_{\alpha \beta} \delta_{i f} .
$$

With Eq. (4) we obtain

$$
\sum_{k}\left\{R_{i k}^{\alpha} R_{R f}^{\beta} E_{k f}-R_{i k}^{\beta} R_{k f}^{\alpha} E_{i k}\right\}=-\frac{\hbar^{2}}{m} \delta_{\alpha \beta} \delta_{i f *}
$$

From the commutator

$$
\left[r_{\alpha}, r_{\beta}\right]=0
$$

we obtain

$$
\sum_{k}\left\{R_{i k}^{\alpha} R_{k f}^{\phi}-R_{i k}^{\alpha} R_{k f}^{\alpha}\right\}=0 .
$$

Multiplying this equation by $\hbar \omega_{1}$ and subtracting the product from Eq. (11) leads to

$$
\begin{aligned}
\sum_{k} & \left\{R_{i k}^{\alpha} R_{k f}^{\beta}\left(E_{k f}-\hbar \omega_{1}\right)-R_{i k}^{\beta} R_{k f}^{\alpha}\left(E_{i k}-\hbar \omega_{1}\right)\right\} \\
& =-\frac{\hbar^{2}}{m} \delta_{\alpha \beta} \delta_{i f} .
\end{aligned}
$$

Conservation of energy implies

$$
E_{i f}=E_{i k}+E_{k f}=\hbar \omega_{1}+\hbar \omega_{2} \text {. }
$$

With this identity, Eq. (14) can be rearranged to

$$
\sum_{k}\left(E_{i k}-h \omega_{1}\right)\left(E_{i k}-h \omega_{2}\right) X_{k_{0} i f}^{\alpha \beta}=\frac{\hbar^{2}}{m} \delta_{\alpha \beta} \delta_{i f} .
$$

Since from Eq. (15) it follows that

$$
\left(E_{i k}-\hbar \omega_{1}\right)\left(E_{i k}-\hbar \omega_{2}\right)=\hbar \omega_{1} \hbar \omega_{2}-E_{i k} E_{k f},
$$

we finally arrive at

$$
\hbar \omega_{1} \hbar \omega_{2} \sum_{k} X_{k_{1} i f}^{\alpha \beta}=\frac{\hbar^{2}}{m} \delta_{\alpha \beta} \delta_{i f}+\sum_{k} E_{i k} E_{k f} X_{k_{1} i f}^{\alpha \beta} .
$$

Equation (18) clearly shows that in the case $i \neq f$ the right-hand sides of Eqs. (5) and (6) are equal if and only if the summation in Eq. (6) includes all the eigenstates of the system. It should be mentioned at this point that this equality no longer holds if a damping factor ${ }^{12}$ is added in the denominator. Since we have found that such a damping factor alters our numerical results by less than $1 \%$ in all cases corresponding to experimentally reasonable conditions $\left(E_{i k}-\hbar \omega_{1}\right.$ $\leq 0.2 \mathrm{eV}$ ), we do not see any need for such factors.

If the initial and the final state are neglected in $\mathrm{Eq}$.
(6) the following error will be introduced:

$$
\Delta \tilde{S}_{\alpha \beta}^{\text {if }}=\frac{1}{\hbar \omega_{1}} R_{i f}^{\beta}\left(R_{f f}^{\alpha}-R_{i 1}^{\alpha}\right)+\frac{1}{\hbar \omega_{2}} R_{i f}^{\alpha}\left(R_{f f}^{\beta}-R_{i 1}^{\beta}\right) .
$$

For a centrosymmetric molecule, $\Delta \tilde{\mathbf{S}}$ is zero since in this case, $\mathbf{R}_{i i}$ and $\mathbf{R}_{f f}$ themselves are zero. Thus, calculations for molecules like hexatriene ${ }^{18}$ do not contain a systematic error, whether $|i\rangle$ and $|f\rangle$ are included or not. For $\Delta \tilde{\mathbf{S}}$ to become important, two conditions have to be fulfilled simultaneously.

(i) The transition $i \rightarrow f$ has to be allowed for one-photon processes $\left(\mathbf{R}_{i f} \neq 0\right)$.

(ii) The difference between the dipole moments of the initial and the final state has to be considerably large.

If we assume $R_{l f}$ to be parallel to $R_{i t}-R_{f f}$, the contribution of $\Delta \tilde{\mathbf{S}}$ to the spatial averaged two-photon cross section for two equally polarized photons of same energy $\left(\delta_{3,}\right)$ becomes

$$
\delta_{+t}=1.043 \frac{f(\Delta \mu)^{2}}{\Delta E}
$$

$f$ is the oscillator strength for the transition $i \rightarrow f, \Delta E$ is the excitation energy (in $\mathrm{eV}$ ), $\Delta \mu$ is the difference between the dipole moments (in Debye), and $\delta$ is measured in $\mathrm{gm}=10^{-50} \mathrm{~cm}^{4} \mathrm{~s}$. The line shape function $g(\omega)$ is set to $5 \times 10^{-15} \mathrm{~s}$, as in Ref. 8. For an intense one-photon allowed transition $(f=1)$ and an excitation energy of $5 \mathrm{eV}$, a change in the dipole moment of 2.3D is sufficient to produce a $\delta$ value of $1 \mathrm{gm}$. From other applications, ${ }^{10,11,16}$ we know that a value of $\delta=1 \mathrm{gm}$ calculated with the same line shape function corresponds to a moderate intense two-photon absorption band in experimental spectra. $\Delta \mu \simeq 3 \mathrm{D}$ is anything but extraordinary for a noncentrosymmetric molecule. The largest values of $\Delta \mu$ known experimentally are in the order of about $15 \mathrm{D},{ }^{19}$ which would lead to a contribution to $\delta(\Delta E=5 \mathrm{eV}, f=1)$ of about $45 \mathrm{gm}$. Such a value corresponds to a very intense TPA band.

\section{APPLICATION TO LINEAR $6 \pi$ SYSTEMS}

To give examples for the theoretical results derived in Sec. II, we now study the following series of compounds:

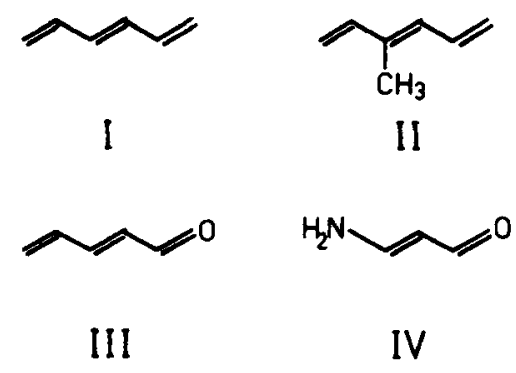

All members of this series possess a linear conjugated $6 \pi$-electron system, but the polarity increases from I to IV. We have selected this series as an example, since longer polyene aldehydes play an important role in the visual process (retinal) and two-photon spectroscopy has been used to study these compounds. ${ }^{12}$ All-trans hexatriene in its ground state has a center 
TABLE I. CNDO-CI results for the polyene model systems I-IV.

\begin{tabular}{|c|c|c|c|c|c|c|c|c|c|}
\hline & & $E^{\mathbf{a}}$ & $\mu^{b}$ & $f^{c}$ & $\delta^{d}$ & $\Omega^{e}$ & $\delta^{*, 2}$ & $\Omega *, \varepsilon$ & $2 D^{b}$ \\
\hline \multirow[t]{8}{*}{ I } & $A_{\varepsilon}^{-}$ & 0.0 & & & & & & & 6.2 \\
\hline & $A_{i}^{-}$ & 40.3 & & & 3.30 & 0.90 & & & 51.8 \\
\hline & $B_{\mu}^{+}$ & 40.9 & & 0.972 & & & & & 2.9 \\
\hline & $B_{u}^{-}$ & 45.8 & & 0.006 & & & & & 40.2 \\
\hline & $\sigma \pi^{*}$ & 54.5 & & & 0.00 & 1.50 & & & 3.1 \\
\hline & $A_{i}^{*}$ & 55.6 & & & 19.20 & 0.66 & & & 9.6 \\
\hline & $\sigma \pi *$ & 61.1 & & & 0.03 & 1.50 & & & 4.1 \\
\hline & $A_{r}^{-}$ & 61.2 & & & 25.08 & 0.73 & & & 74.2 \\
\hline \multirow[t]{8}{*}{ II } & "A $A_{l}^{-"}$ & 0.0 & 0.50 & & & & & & 5.9 \\
\hline & $" A_{c}^{-13}$ & 39.6 & 2.96 & 0.309 & 2.10 & 1.00 & 0.22 & 1.47 & 34.3 \\
\hline & " $B_{u}^{*} "$ & 41.1 & 2.74 & 0.647 & 1.27 & 0.90 & 0.31 & 0.84 & 18.3 \\
\hline & “ $B_{u}^{-7}$ & 45.3 & 0.56 & 0.002 & 0.11 & 0.62 & 0.11 & 0.63 & 40.3 \\
\hline & $\sigma \pi^{*}$ & 54.3 & 0.62 & 0.000 & 0.00 & 1.50 & 0.00 & 1.50 & 3.2 \\
\hline & "A" & 55.0 & 0.51 & 0.002 & 25.86 & 0.65 & 25.90 & 0.65 & 11.3 \\
\hline & $\sigma \pi *$ & 60.6 & 0.82 & 0.000 & 0.05 & 1.50 & 0.01 & 1.50 & 3.7 \\
\hline & "A $\vec{r} "$ & 61.1 & 1.15 & 0.041 & 47.15 & 0.69 & 46.01 & 0.69 & 36.6 \\
\hline \multirow[t]{11}{*}{ III } & $" A_{r}^{-"}$ & 0.0 & 5.26 & & & & & & 4.9 \\
\hline & $n \pi *$ & 26.3 & 1.37 & 0.000 & 0.00 & 1.50 & 0.00 & 1.50 & 10.5 \\
\hline & " $B_{u}^{+\prime "}$ & 41.7 & 9.30 & 0,782 & 7.02 & 0.66 & 0.57 & 0.95 & 5.5 \\
\hline & " $A_{z}^{-"}$ & 44.0 & 3.78 & 0.124 & 1.56 & 1.02 & 0.89 & 1.29 & 44.1 \\
\hline & $n \pi *$ & 44.7 & 1.66 & 0.000 & 0.00 & 1.50 & 0.00 & 1.50 & 39.8 \\
\hline & " $B_{u}^{-7}$ & 51.8 & 5.80 & 0.008 & 3.62 & 0.71 & 3.40 & 0.75 & 36.2 \\
\hline & $\sigma \pi^{*}$ & 53.4 & 4.18 & 0.001 & 0.01 & 1.50 & 0.01 & 1.50 & 15.1 \\
\hline & $\sigma \pi^{*}$ & 56.2 & 2.04 & 0.002 & 0.00 & 1.50 & 0.02 & 1.50 & 53.0 \\
\hline & "As" & 56.9 & 6.41 & 0.004 & 9.92 & 0.67 & 10.06 & 0.68 & 8.0 \\
\hline & $\sigma \pi^{*}$ & 61,9 & 3.08 & 0.000 & 0.03 & 1.50 & 0.03 & 1.50 & 18.1 \\
\hline & " $A_{\vec{E}}^{\vec{r}}$ & 62.9 & 5.74 & 0.057 & 13.05 & 0.72 & 13.64 & 0.72 & 16.6 \\
\hline \multirow[t]{8}{*}{ IV } & "A $A_{p}^{-"}$ & 0.0 & 6.17 & & & & & & 3.2 \\
\hline & $n \pi *$ & 24.7 & 2.91 & 0.000 & 0.00 & 1.50 & 0.00 & 1.50 & 10.0 \\
\hline & " $B_{u}^{+"}$ & 40.6 & 9.63 & 0.513 & 4. 97 & 0.65 & 0.12 & 1.48 & 5.3 \\
\hline & $n \pi^{*}$ & 44.3 & 2.25 & 0.001 & 0.01 & 1.50 & 0.00 & 1.50 & 41.8 \\
\hline & "A $A_{t}^{\vec{r}}$ & 48.8 & 6.48 & 0.019 & 3. 32 & 0.77 & 3.05 & 0.90 & 36.3 \\
\hline & "As" & 56.8 & 6.68 & 0.015 & 0.18 & 0.50 & 0.21 & 0.61 & 14.1 \\
\hline & $\sigma \pi *$ & 57.0 & 3.48 & 0.002 & 0.13 & 1.50 & 0.01 & 1.50 & 8.6 \\
\hline & $\sigma \pi^{*}$ & 60.3 & 5.26 & 0.005 & 1.58 & 1.50 & 1.50 & 1.50 & 4. 7 \\
\hline
\end{tabular}

E: Excitations energies in $1000 \mathrm{~cm}^{-1}$.

b: Dipole moment.

'f: Oscillator strength.

if: Two-photon cross section in $10^{-50} \mathrm{~cm}^{4} \mathrm{~s}$.

' $\Omega$ : Two-photon polarization parameter.

t, The values $\delta^{*}$ and $\Omega^{*}$ have been obtained with the "wrong" formula, omitting the contribution from the initial and final states.

h $q D$ : Contribution of doubly excited configurations in percent.

of symmetry and no dipole moment. Consequently, for the pure vertical electronic transitions, the selection rules are complementary for one- and two-photon ab- sorption, and no contribution may result from $\mathrm{Eq}$. (19) to the two-photon cross section. Substitution of a methyl group in position 3 introduces some polarity and 


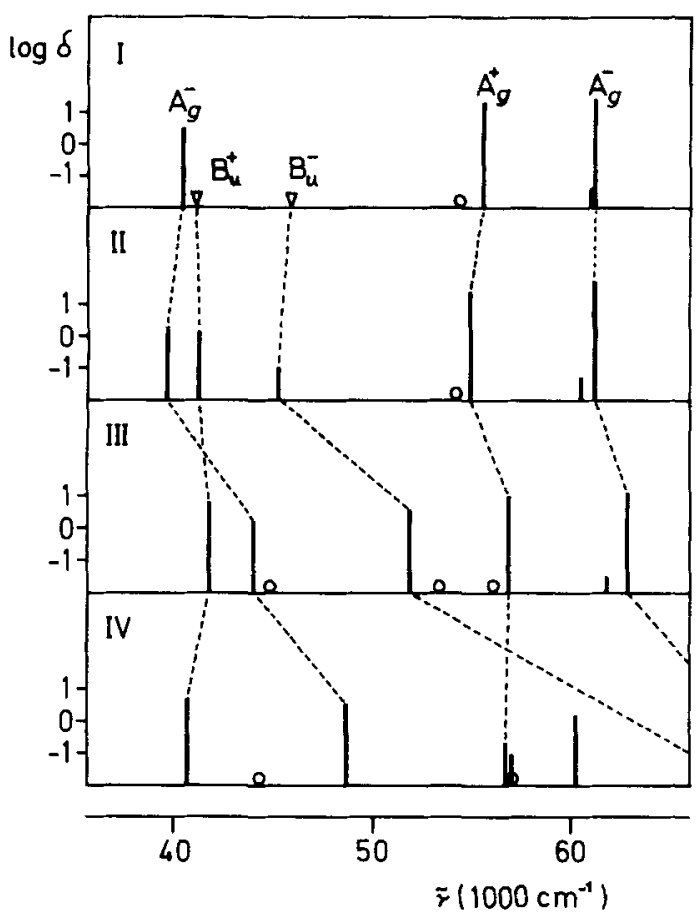

FIG. 1. Correlation diagram for the excited states of the model systems I-IV calculated with CNDO-CI. The lengths of the bars correspond to the calculated two-photon cross sections.

reduces the symmetry from $C_{2 h}$ to $C_{s}$. The polarity is further increased if we go to pentadienal and 3-aminopropenal, the first member of the merocyanine dye class. In this class the dipole moment changes by 10 and more Debye (depending on the length of the conjugated chain) if one goes from the ground to the first excited state.

For these molecules, we have calculated excitation energies, dipole moments of ground and excited states, $f$ values and two-photon absorption cross sections according to the CNDO-CI procedure previously described. 5,8

The Pariser formula is used to approximate the electron repulsion. For the oxygen $\beta_{0}$ parameter a value of $-30.0 \mathrm{eV}$ has been used according to a recent reparametrization of the CNDO/S scheme. ${ }^{20}$ The CI includes the 300 singly and doubly excited configurations of lowest energy. To calculate the two-photon cross sections $\delta$, Eq. (6) has been used, setting both photon energies equal to half the excitation energy $E_{i f}$. This corresponds to the most common single beam experiment, which allows the determination of two independent experimental parameters for each transition namely the cross section for parallel polarized photons $\delta_{1,}$ and the polarization parameter

$$
\Omega=\delta_{\ell d} / \delta_{1+} \text {. }
$$

The results obtained from these calculations are collected in Table I.

In spite of the fact that all $\pi \pi^{*}$ excitations belong to the same irreducible representation in II-IV, the excited states may well be correlated in the whole series
I-IV. This is easily done by inspection of molecular orbitals and of the corresponding eigenvectors of the CI matrix. To indicate from which state in hexatriene the states of II-IV evolve, we use the same symmetry notation for these systems as for hexatriene but put the symbols in quotation marks to remember that the actual symmetry is lower.

With respect to the ground state, the states " $2 A_{\mathrm{g}}^{\text {-") }}$ and

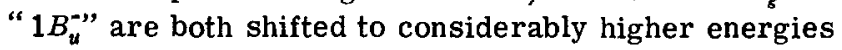
with increasing polarity (Fig. 1). Both states correspond to covalent states in VB theory, ${ }^{2}$ states which become energetically less favorable in an inherently polar molecule. The strong mixing with doubly excited configurations which stabilize these states with respect to the plus states in an MO description of polyenes ${ }^{2}$ is therefore reduced with increasing polarity (see Table I, column 9).

The only transition below $7 \mathrm{eV}$ with high calculated oscillator strength is " $1 A_{g}^{-"} \rightarrow$ " $1 B_{u}^{+, "}$ in all four molecules. In II and III, considerable intensity is also calculated for " $1 A_{\frac{-}{s}}^{-}$- " $2 A_{g}^{-}$," but since both transitions lie close together in these molecules, the one-photon spectra of I-IV are predicted to be very similar with only one intense band around $5 \mathrm{eV}$. This is in good agreement with the experimental spectra (compare data given in Table II). The energy of the low lying $n \pi^{*}$ transition appearing in III and IV is also reproduced reasonably well in our calculations.

Two intense two-photon transitions are calculated for hexatriene below $7 \mathrm{eV}$ (Fig. 1):

$$
1 A_{g}^{-}-2 A_{g}^{-}(\delta=3.30) \text { and } 1 A_{g}^{-}-1 A_{g}^{+}(\delta=19.2) \text {. }
$$

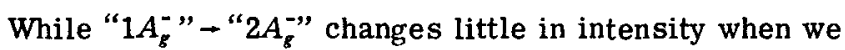

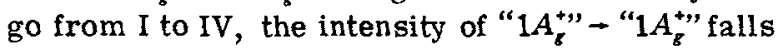
rapidly in this series. Contrary to this, the transitions " $1 A_{g}^{-", ~-~ " ~} 1 B_{u}^{+"}$ and " $1 A_{g}^{-", ~-~ " ~} 1 B_{u}^{-",}$, which are symmetry forbidden in hexatriene, gain considerable intensity with increasing polarity. In III and IV, " $1 A_{g}^{-, "-~ " ~} 1 B_{u}^{+}$," the transition mainly responsible for the intense one-photon absorption around $5 \mathrm{eV}$ is predicted to have a larger two-photon cross section than " $1 A_{g}^{-"} \rightarrow$ " $2 A_{g}^{-}$"" Thus, we have to be aware that we may find two two-photon allowed transitions at the beginning of the $\pi \pi^{*}$ spectrum instead of one as in the parent polyene. We also learn from these simple $6 \pi$ systems that it is not possible to conclude from the observation of a twophoton allowed transition in the low energy part of the $\pi \pi^{*}$ spectrum of a polyenealdehyde like retinal ${ }^{12}$ that the final state of this transition corresponds to the famous low lying $A_{g}^{-}$state of the polyenes. Even such a small perturbation as a methyl group seems to introduce enough polarity to the system to make the first one-photon allowed $\pi \pi^{*}$ transition visible in the twophoton spectrum.

Let us finally discuss the importance of the initial and the final state for the calculated two-photon intensities. If we neglect these states as intermediate states in our calculation the $\delta^{*}$ values shown in column 7 of Table $I$ are found. As it had to be expected from the analysis of Eq. (19), the inclusion of $|i\rangle$ and $|f\rangle$ is only important for those states of the polar systems 
TABLE II. Experimental band maxima (in $1000 \mathrm{~cm}^{-1}$ ) for some compounds containing the $\pi$ electron system of the model compounds I-IV.

\begin{tabular}{|c|c|c|c|c|}
\hline Molecule & $\begin{array}{l}\text { Band maximum } \\
\left(1000 \mathrm{~cm}^{-1}\right)\end{array}$ & Solvent & $\epsilon(1 / \mathrm{mol} \mathrm{cm})$ & Reference \\
\hline & 39.8 & gas & 21000 & a \\
\hline & $\begin{array}{l}29.4 \\
38.4\end{array}$ & $A^{2}$ & $\begin{array}{r}43 \\
25000\end{array}$ & b \\
\hline & 37.7 & $C^{\mathrm{h}}$ & 28950 & c \\
\hline & 37.5 & $C$ & 22400 & c \\
\hline & 34.5 & $A$ & & $\mathrm{~d}$ \\
\hline & 34.8 & $M^{\mathrm{R}}$ & 28000 & e \\
\hline
\end{tabular}

${ }^{2}$ R. M. Gavin and S. A. Rice, J. Chem. Phys, 60, 3231 (1974).

bMS-UV Atlas (Butterworths, London, 1971), Spectrum No. B4/1.

'A. F. Kluge and C. P. Lillya, J. Org. Chem. 36, 1977 (1971).

dP. J. Brignel, U. Eisner, and P. G. Farrell, J. Chem. Soc. B 1966, 1083.

D. L. Ostercamp, J. Org. Chem. 35, 1632 (1970).

'A: Ethanol.

$M$ : Methanol.

${ }^{n} \mathrm{C}$ : Cyclohexane.

to which a one-photon excitation is allowed. In our examples, these are the states denoted " $1 B_{u}^{+ \text {" }}$ and " $2 A_{g}^{-}$." For all the other states given in Table I the difference between $\delta^{*}$ and $\delta$ is negligible. Most of the calculated intensity $(92 \%-98 \%)$ of the two-photon transition " $1 A_{e}^{- \text {" }}$ - " $1 B_{u}^{+"}$ results, however, from the inclusion of the initial and the final state. Neglect of these two states as intermediate states would lead to the prediction of very little two-photon intensity for the one-photon allowed " $1 A_{z}^{-}-$" $1 B_{u}^{+"}$ transition. The intensity ratio $\delta\left(B_{u}^{+}\right) / \delta\left(2 A_{z}^{-}\right)$ is changed from 4.5 to 0.64 for III and from 1.5 to 0.04 for IV if we neglect $\Delta \tilde{s}[\mathrm{Eq} .(19)]$. Since intensity ratios should be measured more easily than absolute values an experimental proof of the importance of $|i\rangle$ and $|f\rangle$ as intermediate states is well within the limit of presently available experimental techniques.

\section{CONCLUSIONS}

From the formalistic point of view we have to state the following:

(i) For molecules having nonvanishing dipole moments, the initial and the final state have to be included in the summation over virtual intermediate states if two-photon cross sections are to be calculated according to Eq. (6). Use of this formula instead of formula (5) is highly favored by its better convergence behavior. The contribution of $|i\rangle$ and $|f\rangle$ to Eq. (6) depends on the product of the one-photon transition moment and the change of dipole moment associated with the excita- tion. Contrary to the Raman process ${ }^{17}$ the contributions of $|i\rangle$ and $|f\rangle$ may become important in two-photon absorption since electronic excitations can lead to considerable changes in the dipole moment.

If we look at our results from a more physical point of view, we can extract from (i) the following statement:

(ii) One-photon allowed transitions will most likely also show non-negligible two-photon intensities if the excitation leads to a change in dipole moment, as it usually does in polar molecules.

From this we finally conclude:

(iii) The detection of a two-photon allowed transition in a polar molecule yields no evidence at all that this transition is connected with a two-photon allowed but one-photon forbidden transition in the parent nonpolar hydrocarbon as it has been proposed for retinol. ${ }^{12}$

\section{ACKNOWLEDGMENTS}

We thank the "Deutsche Forschungsgemeinschaft" and the "Regionales Rechenzentrum Köln" for financial support respective providing the necessary computer time.

${ }^{1} J$. Koutecky, J. Chem. Phys. 47, 1501 (1967).

${ }^{2}$ K. Schulten, I. Ohmine, and M. Karplus, J. Chem. Phys. 64, 4422 (1976). 
${ }^{3}$ P. Tavan and K. Schulten, J. Chem. Phys. 70, 5414 (1979).

"Following the definition given in Ref. 5 we use the abbreviation "dipole forbidden" not only for transitions which are strictly forbidden by symmetry, but also for those for which the transition dipole moment is usually small due to the action of other less strict selection rules.

${ }^{5}$ B. Dick and G. Hohlneicher, Theor. Chim. Acta (Berlin) 53, 221 (1979).

${ }^{6}$ W. M. McClain, J. Chem. Phys, 55, 2789 (1971).

${ }^{7}$ W. M. Mcclain, Acc. Chem. Res, 7, 129 (1974).

${ }^{8}$ G. Hohlneicher and B. Dick, J. Chem. Phys. 70, 5427 (1979).

${ }^{9} \mathrm{~B}$. Honig and J. Jortner, J. Chem. Phys. 47, 3698 (1967).

${ }^{10}$ B. Dick and G. Hohlneicher, Chem. Phys. Lett. 83, 615 (1981).

${ }^{11}$ B. Dick and G. Hohlneicher, Chem. Phys. Lett. 84, 471 (1981).

${ }^{12}$ R. R. Birge, J. A. Bennett, B. M. Pierce, and T. M. Thomas, J. Am. Chem. Soc. 100, 1533 (1978).

${ }^{13}$ R. R. Birge and B. M. Pierce, J. Chem. Phys. 70, 165 (1979).

${ }^{14}$ B. T. Marchese, C. J. Seliskar, and H. H. Jaffé, J. Chem. Phys. 72, 4194 (1980).

${ }^{15}$ After completion of this work (Ref. 16) an article by Marten- sen and Svendsen appeared in this journal (Ref. 17) in which a corresponding problem is discussed, with respect to Raman scattering. Here a similar confusion concerning initial and final states as intermediate states has been found in the literature. Since both Raman scattering and two-photon absorption are two-photon processes, the basic theoretical results of Martensen and Svendsen are similar to those derived in Sec. II. The final result, however, is quite different. In Raman scattering the neglect of initial and final states as intermediate states is (according to Martensen and Svendsen) of little importance, whereas, in two-photon absorption these states may be responsible for the leading terms in the calculation of the cross section.

${ }^{16} \mathrm{~B}$. Dick, thesis, Universität du Köln, Köln, 1981 .

${ }^{17}$ O. S. Martensen and E. N. Svendsen, J. Chem. Phys. 74, 3185 (1981).

${ }^{18}$ A. J. Twarowski and D. S. Kliger, Chem. Phys, Lett. 50, 36 (1977).

${ }^{19}$ W. Liptay, in Excited States, edited by E. C. Lim (Academic, New York, 1974), Vol. I.

${ }^{20}$ (a) H. H. Jaffé (private communication); (b) P. Jaques, J. Faure, O. Chalvet, and H. H. Jaffé, J. Phys. Chem. 85, $473(1981)$. 